\title{
Synchronization of spiral wave patterns in two-layer 2D lattices of nonlocally coupled discrete oscillators
}

Cite as: Chaos 29, 053105 (2019); https://doi.org/10.1063/1.5092352

Submitted: 11 February 2019 . Accepted: 12 April 2019. Published Online: 07 May 2019

A. V. Bukh, E. Schöll (D), and V. S. Anishchenko
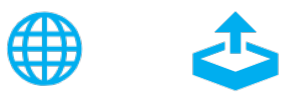

Export Citation

\section{ARTICLES YOU MAY BE INTERESTED IN}

Complexity reduction ansatz for systems of interacting orientable agents: Beyond the Kuramoto model

Chaos: An Interdisciplinary Journal of Nonlinear Science 29, 053107 (2019); https:// doi.org/10.1063/1.5093038

Spike chimera states and firing regularities in neuronal hypernetworks

Chaos: An Interdisciplinary Journal of Nonlinear Science 29, 053115 (2019); https: // doi.org/10.1063/1.5088833

Collective dynamics of globally delay-coupled complex Ginzburg-Landau oscillators Chaos: An Interdisciplinary Journal of Nonlinear Science 29, 053104 (2019); https:// doi.org/10.1063/1.5087188

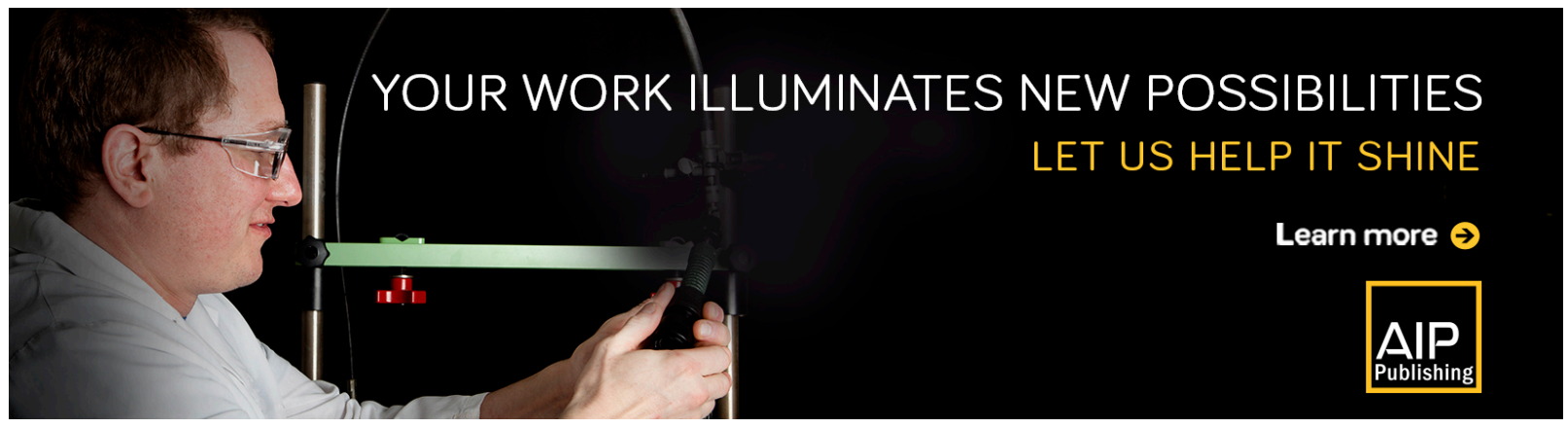




\title{
Synchronization of spiral wave patterns in two-layer 2D lattices of nonlocally coupled discrete oscillators
}

\author{
Cite as: Chaos 29, 053105 (2019); doi: 10.1063/1.5092352 \\ Submitted: 11 February 2019 . Accepted: 12 April 2019. \\ Published Online: 7 May 2019

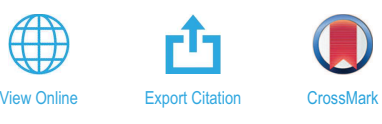

A. V. Bukh, ${ }^{1, a)}$ E. Schöll, ${ }^{2, b)}$ (iD and V. S. Anishchenko ${ }^{1, c)}$

\author{
AFFILIATIONS \\ ${ }^{1}$ Department of Physics, Saratov State University, Astrakhanskaya str. 83, Saratov 410012, Russia \\ ${ }^{2}$ Institut für Theoretische Physik, Technische Universität Berlin, Hardenbergstraße 36, Berlin 10623, Germany \\ Electronic mail: buh.andrey@yandex.ru

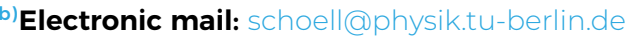 \\ ${ }^{c}$ Electronic mail: wadim@info.sgu.ru
}

\begin{abstract}
The paper describes the effects of mutual and external synchronization of spiral wave structures in two coupled two-dimensional lattices of coupled discrete-time oscillators. Each lattice is given by a 2D $N \times N$ network of nonlocally coupled Nekorkin maps which model neuronal activity. We show numerically that spiral wave structures, including spiral wave chimeras, can be synchronized and establish the mechanism of the synchronization scenario. Our numerical studies indicate that when the coupling strength between the lattices is sufficiently weak, only a certain part of oscillators of the interacting networks is imperfectly synchronized, while the other part demonstrates a partially synchronous behavior. If the spatiotemporal patterns in the lattices do not include incoherent cores, imperfect synchronization is realized for most oscillators above a certain value of the coupling strength. In the regime of spiral wave chimeras, the imperfect synchronization of all oscillators cannot be achieved even for sufficiently large values of the coupling strength.
\end{abstract}

Published under license by AIP Publishing. https://doi.org/10.1063/1.5092352

Synchronization, being one of the fundamental nonlinear phenomena of nature, continues to play an important role in the modern research in the natural sciences. Many important problems have been considered and solved when studying synchronization of coupled oscillators, including chaotic ones. Nowadays, special interest is targeted on the synchronization of interacting networks, which exhibit a rich variety of complex spatiotemporal patterns. The present research is devoted to the study of twodimensional spiral autowave structures. These were previously observed in biological experiments and numerical simulations of neural networks of the brain and human cardiac fibrillations in cardiology. We explore numerically the effects of mutual and external synchronization of spiral wave structures, including spiral wave chimeras, in a system of two coupled $2 \mathrm{D}$ lattices of nonlocally coupled discrete maps and reveal the peculiarities of these phenomena. The discrete Nekorkin map, that simulates the neural activity, is selected as an individual oscillator in the networks. It is shown that with increasing coupling strength between the lattices, the number of synchronized elements gradually grows and the imperfect synchronization is observed for the case of relatively simple spatiotemporal structures, which do not include incoherent cores. Only partial synchronization takes place when the networks exhibit spiral wave chimera structures. In this case, oscillators in coherent domains are imperfectly synchronized, while elements in incoherent cores remain desynchronized. Complete in-phase synchronization is not achieved even if the coupling strength increases considerably.

\section{INTRODUCTION}

Formation and evolution of various dissipative structures in complex ensembles of interacting oscillators represent one of the key research directions in modern natural science. ${ }^{1-9}$ In the last 15 years, the attention of specialists in nonlinear dynamics and related scientific fields was focused on the studies of socalled "chimera states." $10-13$ These states are characterized by the coexistence of clusters of oscillators with coherent (synchronous) 
and incoherent (asynchronous) dynamics in the ensemble space. Of particular interest is the synchronization of chimera states in multicomponent systems and networks. Recently, various synchronization scenarios in multiplex and multilayer ${ }^{14-22}$ networks were studied, such as generalized synchronization, ${ }^{23}$ interlayer (external and mutual) synchronization, ${ }^{24-29}$ relay (remote) synchronization, ${ }^{30,31}$ and explosive synchronization. ${ }^{32-35}$ Among a large variety of chimera states, ${ }^{26,36-63}$ here we concentrate on so-called "spiral wave chimera structures," which are observed in 2D ensembles of coupled nonlinear oscillators. ${ }^{13,64-79}$ These were typically found in the case of nonlocal coupling topology of network elements. These structures represent spiral waves which rotate around incoherent cores. The network elements in the regions of rotating spiral waves are characterized by coherent dynamics, while the elements inside the incoherent cores oscillate asynchronously. We note that spiral wave chimeras were observed in both numerical and experimental ${ }^{70}$ studies of the dynamics of 2D ensembles whose individual oscillators are described, as a rule, by systems of ordinary differential equations. The model of a 2D lattice consisting of nonlocally coupled discrete-time oscillators which describe neural activity ${ }^{80}$ has been first proposed and explored in Ref. 79. It was shown that this discrete model of the 2D lattice can exhibit all typical spiral wave structures, including spiral wave chimeras, which were found earlier for networks of coupled continuous-time systems. ${ }^{13,65-73,77,78}$ However, effects of synchronization of spiral wave chimera structures have not been studied yet. In the present paper, we describe numerical results for mutual and external synchronization of spiral wave structures in two coupled $2 \mathrm{D}$ lattices consisting of nonlocally coupled discrete-time neuronal models. $^{79,80}$

\section{MODELS UNDER STUDY}

\section{A. Single Nekorkin map}

Before focusing on the study of two coupled 2D lattices, we describe briefly the dynamics of a single Nekorkin map which is a simple model for neuronal dynamics. ${ }^{80}$ It is defined by the following equations:

$$
\begin{aligned}
& x^{t+1}=x^{t}+F\left(x^{t}\right)-y^{t}-\beta H\left(x^{t}-d\right), \\
& y^{t+1}=y^{t}+\varepsilon\left(x^{t}-J\right),
\end{aligned}
$$

where $x^{t}$ is a variable that describes the dynamics of the membrane potential of the nerve cell, $y^{t}$ is a variable that relates to the cumulative effect of all ion currents across the membrane, and functions $F\left(x^{t}\right)$ and $H\left(x^{t}-d\right)$ are given as follows:

$$
\begin{aligned}
& F\left(x^{t}\right)=x^{t}\left(x^{t}-a\right)\left(1-x^{t}\right), \quad 0<a<1, \\
& H\left(x^{t}\right)= \begin{cases}1, & x^{t}>0, \\
0 & \text { elsewhere. }\end{cases}
\end{aligned}
$$

The parameter $\varepsilon>0$ determines the characteristic time scale of $y^{t}$, the parameter $J$ controls the level of the membrane depolarization $(J<d)$, and the parameters $\beta>0$ and $d>0$ determine the excitation threshold of bursting oscillations, $t=1,2, \ldots$, represents discrete time. Despite its simplicity, this map can describe a number of basic modes of neuronal activity ${ }^{81}$ when the control parameters are
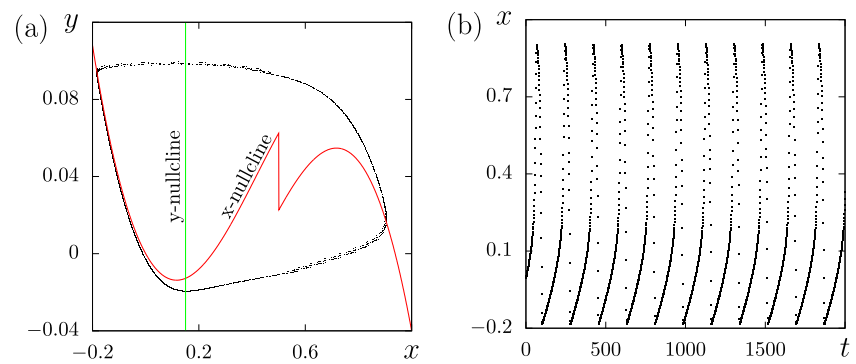

FIG. 1. (a) Phase portrait and (b) time series $x^{t}$ for the map (1) at $a=0.25, \beta=$ $0.04, J=0.15, d=0.5$, and $\varepsilon=0.005$. The Lyapunov exponents are $\Lambda_{0}=0.0$ and $\Lambda_{1}=-0.4$, and the rotation number is $r=0.014$.

varied. These modes include spike-bursting chaotic oscillations, subthreshold oscillations, as well as the regime of single, periodic, and chaotic spike generation. ${ }^{8}$

In our studies, we are especially interested in the dynamical regime of the map (1), which relates to spike oscillations. This mode is exemplified in Fig. 1, where the phase portrait and time series for the variable $x^{t}$ are plotted, respectively. The corresponding phase portrait represents a closed invariant curve. The maximal Lyapunov exponent in this regime is (up to numerical inaccuracy) zero, and the second one is negative. Thus, we can conclude that map (1) dynamics reflects a quasiperiodic mode in a lifted continuous-time system. ${ }^{82}$ However, as clearly seen from Fig. 1(b), the time series of the variable $x^{t}$ is nearly periodic, and the rotation number for the invariant curve [Fig. 1(a)] is very small, i.e., $r=0.014$. In this case, the trajectory shifts on a very small angle per iteration that leads to the observation of nearly periodic oscillations, and the invariant curve is very similar to a limit cycle. This fact is also confirmed by numerical results for the autocorrelation function. ${ }^{79}$ The latter decays gradually and very slowly as a consequence of nearly periodic oscillations.

\section{B. 2D lattice of coupled Nekorkin maps}

In Ref. 79, Nekorkin map (1) was chosen as the individual element in a two-dimensional $N \times N$ lattice of nonlocally coupled oscillators. The network equations are as follows:

$$
\begin{aligned}
x_{i, j}^{t+1}= & x_{i, j}^{t}+F\left(x_{i, j}^{t}\right)-y_{i, j}^{t}-\beta H\left(x_{i, j}^{t}-d\right) \\
& +\frac{\sigma_{x}}{B_{i, j}^{x}} \sum_{m_{x}, n_{x}}\left[f\left(x_{m_{x}, n_{x}}^{t}\right)-f\left(x_{i, j}^{t}\right)\right], \\
y_{i, j}^{t+1}= & y_{i, j}^{t}+\varepsilon\left(x_{i, j}^{t}-J\right),
\end{aligned}
$$

where $m_{x}, n_{x} \in \mathbb{N}$ are indices for nonlocal neighbors. The sum denotes nonlocal coupling of range $R_{x}$ in a square domain. Network (4) was analyzed for both periodic and no-flux boundary conditions in Ref. 79. The double index of variables $x_{i, j}$ and $y_{i, j}$ with $i, j=1, \ldots, N$ encodes the position of corresponding oscillators on the two-dimensional lattice. The parameter $\sigma_{x}$ denotes the coupling strength between the elements in the $x$ variable, and $B_{i, j}^{x}$ gives the number of nonlocally coupled neighbors of node $(i, j)$. In the case of periodic boundary conditions, we have $B_{i, j}^{x}=\left(2 R_{x}+1\right)^{2}-1$. In 
the case of no-flux ${ }^{83}$ boundary conditions, $B_{i, j}^{x}$ satisfies the following relations:

$$
\left\{\begin{array}{l}
\max \left(1, i-R_{x}\right) \leqslant m_{x} \leqslant \min \left(N, i+R_{x}\right), \\
\max \left(1, j-R_{x}\right) \leqslant n_{x} \leqslant \min \left(N, j+R_{x}\right) .
\end{array}\right.
$$

The numerical results described in Ref. 79 have shown that when the nonlocal coupling strength $\sigma_{x}$ and the coupling range $R_{x}$ are varied, model (4) can demonstrate all the typical spiral wave patterns, including spiral wave chimeras, which were observed earlier.

\section{Two coupled 2D lattices of Nekorkin maps}

We now couple two 2D lattices each described by network (4) of $200 \times 200$ nonlocally coupled Nekorkin maps. The coupling between the lattices is assumed to be mutual. This means that only corresponding oscillators of the lattices are mutually coupled via their coordinates, i.e., in a multiplex configuration. In this case, the coupled lattices are described by the following system of equations:

$$
\begin{aligned}
x_{i, j}^{t+1}= & x_{i, j}^{t}+F\left(x_{i, j}^{t}\right)-y_{i, j}^{t}-\beta H\left(x_{i, j}^{t}-d\right) \\
& +\frac{\sigma_{x}}{B_{i, j}^{x}} \sum_{m_{x}, n_{x}}\left[f\left(x_{m_{x}, n_{x}}^{t}\right)-f\left(x_{i, j}^{t}\right)\right]+\gamma_{u x}\left[u_{i, j}^{t}-x_{i, j}^{t}\right], \\
y_{i, j}^{t+1}= & y_{i, j}^{t}+\varepsilon\left(x_{i, j}^{t}-J\right), \\
u_{i, j}^{t+1}= & u_{i, j}^{t}+F\left(u_{i, j}^{t}\right)-v_{i, j}^{t}-\beta H\left(u_{i, j}^{t}-d\right) \\
& +\frac{\sigma_{u}}{B_{i, j}^{u}} \sum_{m_{u}, n_{u}}\left[f\left(u_{m_{u}, n_{u}}^{t}\right)-f\left(u_{i, j}^{t}\right)\right]+\gamma_{x u}\left[x_{i, j}^{t}-u_{i, j}^{t}\right], \\
v_{i, j}^{t+1}= & v_{i, j}^{t}+\varepsilon\left(u_{i, j}^{t}-J\right),
\end{aligned}
$$

where variables $x_{i, j}^{t}, y_{i, j}^{t}$ define the dynamics of the oscillators in the first lattice, variables $u_{i, j}^{t}, v_{i, j}^{t}$ determine the dynamics of the oscillators in the second lattice, and $\gamma_{u x}, \gamma_{x u}$ are the interlattice coupling strengths between corresponding oscillators of the first and second lattice layer. In our studies, we consider only the case of no-flux boundary conditions. Link indices $m_{x}, n_{x} \in \mathbb{N}$ are given in Eq. (5) for the first lattice, and indices $m_{u}, n_{u} \in \mathbb{N}$ in the second lattice are defined analogously by replacing $R_{x}$ with $R_{u}$. To account for potentially different coupling parameters in both networks, we introduce a subscript $x$ and $u$ for the first and second network in the coupling strengths $\sigma_{x}, \sigma_{u}$, coupling ranges $R_{x}, R_{u}$, numbers of nonlocal neighbors $B_{i, j}^{x}, B_{i, j}^{u}$, and neighbor indices $m_{x}, m_{u}, n_{x}, n_{u}$. The values of the coupling strengths are fixed as $\sigma_{x}=\sigma_{u}=0.6$.

The synchronization of oscillations between the lattices is quantified by calculating the number of synchronized elements $N_{s}$ in the lattices, which satisfy the condition $r_{i, j} \geqslant 0.95$, where the correlation coefficient $r_{i, j}$ between corresponding oscillators of the lattices is given as follows:

$$
\begin{aligned}
r_{i, j} & =\frac{\left\langle\tilde{x}_{i, j} \tilde{u}_{i, j}\right\rangle}{\sqrt{\left\langle\tilde{x}_{i, j}^{2}\right\rangle\left\langle\tilde{u}_{i, j}^{2}\right\rangle}}, \\
\tilde{x}_{i, j} & =x_{i, j}-\left\langle x_{i, j}\right\rangle, \tilde{u}_{i, j}=u_{i, j}-\left\langle u_{i, j}\right\rangle .
\end{aligned}
$$

The correlation coefficient $r_{i, j}$ is widely used when synchronization of coupled oscillators is studied. ${ }^{51,84}$ The corresponding oscillators are
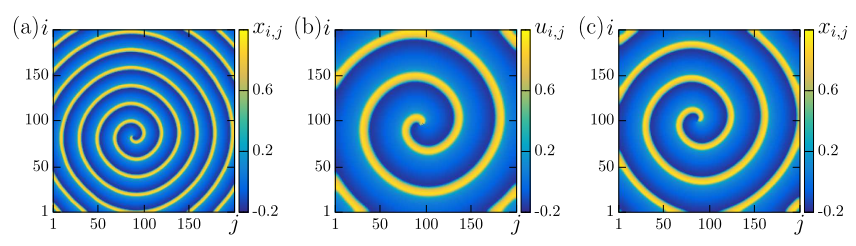

FIG. 2. Snapshots of (a) $x_{i, j}$ (first lattice) for $R_{x}=1, \gamma=0$, (b) $u_{i, j}$ (second lattice) at $R_{u}=3, \gamma=0$, and (c) $x_{i, j}$ (first lattice) for $\gamma=0.04$. Other parameters as in Fig. 1.

assumed to be synchronized if $r_{i, j} \geqslant 0.95$, otherwise they are desynchronized. The threshold value of $r_{i, j}^{\text {th }}=0.95$ is chosen because complete synchronization cannot be achieved in the case of parameter detuning in the interacting lattices. The condition $r_{i, j} \geqslant 0.95$ characterizes the maximum degree of synchronization of spatiotemporal structures, which is possible in the regimes studied.

\section{NUMERICAL RESULTS FOR MUTUAL SYNCHRONIZATION}

The parameters of individual map (1) are set as in Sec. II. The coupling strengths between the lattices are assumed to be $\gamma_{u x}=$ $\gamma_{x u}=\gamma$ in the case of mutual synchronization. We consider the dynamics of coupled lattices (6) when the coupling strength $\gamma$ is varied. In our simulations, spatiotemporal patterns in network (4) are obtained as follows. We use random initial conditions distributed in the intervals $x_{i, j}^{0}, u_{i, j}^{0} \in[-0.2,0.6], y_{i, j}^{0}, v_{i, j}^{0} \in[-0.02,0.06]$ for the coupling range $R_{x}=R_{u}=1$. Whenever we observe a spiral wave pattern in each uncoupled lattice at $R_{x}=R_{u}=1$, we use this as initial condition and continue our calculation by changing the coupling parameters. First, we choose simple but nonidentical spiral wave structures which are realized in the lattices when they are uncoupled. These patterns are exemplified in Figs. 2(a) and 2(b). As can be seen from Fig. 2(c), when the mutual coupling $\gamma$ is turned on, the resulting structure in network (6) differs from the initial patterns in both lattices [Figs. 2(a) and 2(b)] and represents a certain intermediate regime due to the mutual coupling which is invasive. The topology of this spiral wave structure is preserved, but the wavelength of the spiral wave in Fig. 2(c) does not coincide with that of the initial structures [Figs. 2(a) and 2(b)]. We measure the wavelength for each
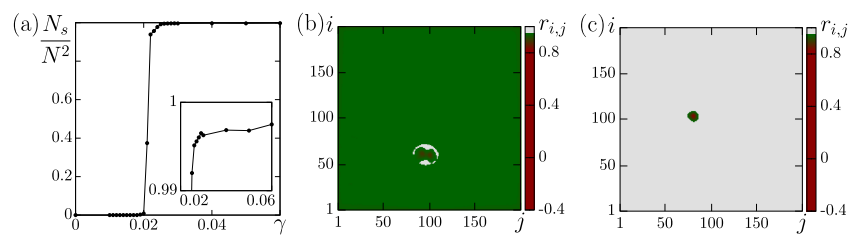

FIG. 3. (a) Number of synchronous oscillators $\left(\left\{N_{s}: r_{i, j}>0.95\right\}\right)$ vs the interlattice coupling strength $\gamma$, distribution of the correlation coefficient $r_{i, j}$ at (b) $\gamma=0.02$ and (c) $\gamma=0.04$ with $R_{x}=1, R_{u}=3$. Coherent oscillators $(i, j)$ are marked by the light tone (gray) and incoherent ones by the dark tone (green or red). The inset in (a) shows a blowup of $N_{s} / N^{2}$ close to unity. 

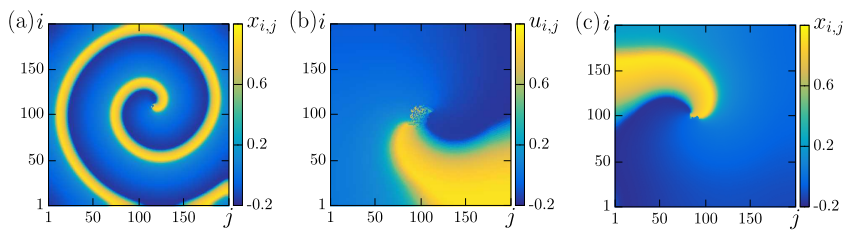

FIG. 4. Snapshots of (a) $x_{i, j}$ (first lattice) for $R_{x}=4, \gamma=0$, (b) $u_{i, j}$ (second lattice) at $R_{u}=22, \gamma=0$, and (c) $x_{i, j}$ (first lattice) for $\gamma=0.06$.

pattern by taking a cross section through the spiral center and then compare the wavelengths.

Our calculations show that different oscillators in the lattices are synchronized at different values of the interlattice coupling strength $\gamma$. As follows from Fig. 3(a), when the coupling strength is rather weak, $\gamma \leq 0.02$, none of the oscillators $\left(N_{s}=0\right)$ are in-phase synchronized. This is well illustrated by the distribution of the correlation coefficient $r_{i, j}$ shown in Fig. 3(b). Synchronization is observed only with $\gamma>0.02$, and most oscillators are synchronized at $\gamma \simeq$ 0.03 . However, the distribution of the correlation coefficient $r_{i, j}$ depicted in Fig. 3(c) clearly indicates that there are a certain number of desynchronized oscillators, which are located in the center of the lattices. For these oscillators, the correlation coefficient $r_{i, j}<0.9$. Hence, we can state that partial synchronization takes place in this case. The regime of partial synchronization is characterized by the coexistence of synchronous and asynchronous oscillators in the interacting lattices. As can be seen from Figs. 2(c) and 3(c), the asynchronous oscillators correspond to the core of the spiral wave. Almost complete synchronization of all elements is achieved when $\gamma>0.05$.

We now consider mutual synchronization of a coherent spiral wave and a spiral wave chimera with single core pictured in Fig. 4. Numerical calculations show that the topology of spiral wave structures essentially depends on the coupling ranges $R_{x}$ and $R_{u}$. Particularly, the number of incoherent cores changes when $R_{x}$ and $R_{u}$ are varied. When the lattices are uncoupled, a spiral wave pattern is realized in the first lattice [Fig. 4(a)], and a spiral wave chimera is observed in the second one [Fig. 4(b)]. The synchronous structure which results from the mutual synchronization of the two coupled lattices (6) is presented in Fig. 4(c). Our numerical results indicate that the effect of partial synchronization manifests itself more brightly in this case. Moreover, as follows from Fig. 5(a), a larger interlattice coupling strength $\gamma \gtrsim 0.04$ is needed to synchronize most of the oscillators in the lattices. At the same time, the number
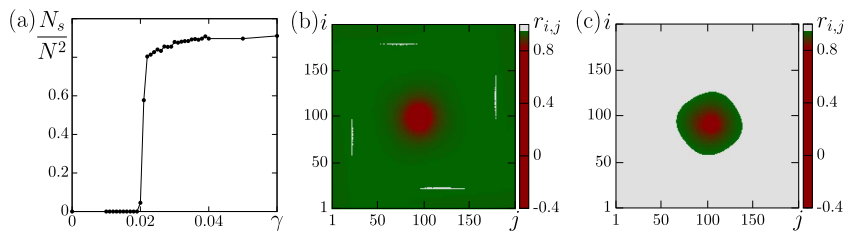

FIG. 5. (a) Number of synchronous oscillators $\left(\left\{N_{s}: r_{i j}>0.95\right\}\right)$ in dependence on the interlattice coupling strength $\gamma$, distribution of the $r_{i, j}$ values at (b) $\gamma=0.02$ and (c) $\gamma=0.06$ with $R_{x}=4, R_{u}=22$.
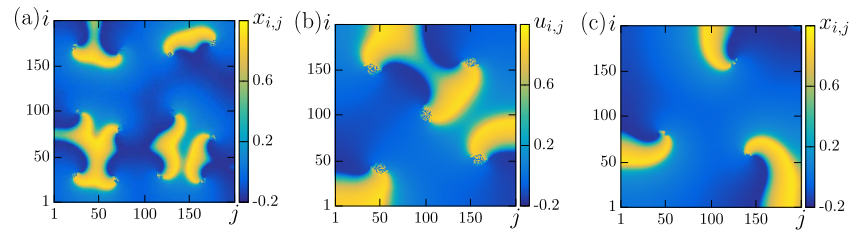

FIG. 6. Snapshots of (a) $x_{i, j}$ (first lattice) for $R_{x}=6, \gamma=0$, (b) $u_{i, j}$ (second lattice) at $R_{u}=14, \gamma=0$, and (c) $x_{i, j}$ (first lattice) for $\gamma=0.08$.

of oscillators which remain desynchronized increases [Fig. 5(c)] as compared to Fig. 3(c). The distribution of the correlation coefficient $r_{i, j}$ values for the transient structure at $\gamma=0.02$ is shown in Fig. 5(b). A comparison of the results presented in Figs. 4-7 shows that the number of desynchronized oscillators increases together with the number of incoherent cores.

To further investigate this observation, we choose multicore spiral wave chimera structures in both lattices when they are uncoupled. They are illustrated in Figs. 6(a) and 6(b) and represent multicore spiral wave chimeras with a different number of incoherent cores in each lattice. However, as seen from Fig. 6(c), the mutual coupling between the lattices simplifies the resulting synchronous structure as compared with the initial ones. It includes only three incoherent cores when the interlattice coupling strength is sufficiently strong $(\gamma>0.05)$. As before (Figs. 4 and 5), in the regime of spiral wave chimera with several incoherent cores, a stronger interlattice coupling strength $\gamma>0.08$ is required to synchronize most of the oscillators [Fig. 7(a)]. The number of desynchronized oscillators for which the condition $r_{i, j}>0.95$ does not hold becomes larger [Fig. 7(c)], and, as follows from Fig. 7(a), the almost complete synchronization of most oscillators in the coupled lattices is not achieved. It is worth noting that the $N_{s}(\gamma)$ dependence becomes smoother when the uncoupled lattices exhibit multicore spiral wave chimeras [compare Figs. 3(a) and 3(b), 5(a) and 5(b), and 7(a) and 7(b)].

\section{NUMERICAL RESULTS FOR EXTERNAL SYNCHRONIZATION}

We now analyze the case of external synchronization when the interlattice coupling is introduced unidirectionally from the elements of the second lattice, which is the driver network, to the corresponding elements of the first lattice, which is the response network. Thus, we set $\gamma_{u x}=\gamma, \gamma_{x u}=0$ in (6). The results of
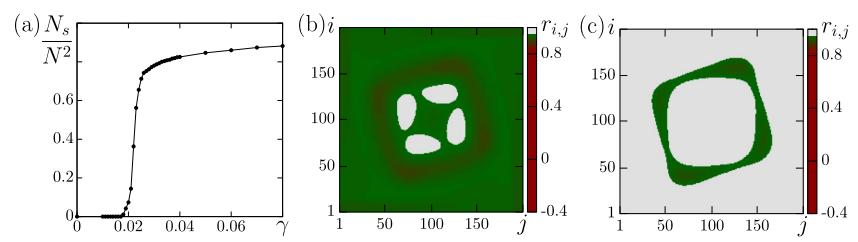

FIG. 7. (a) Number of synchronized oscillators $\left(\left\{N_{s}: r_{i, j}>0.95\right\}\right)$ vs the interlattice coupling strength $\gamma$, distribution of the $r_{i, j}$ values at (b) $\gamma=0.02$ and (c) $\gamma=0.08$ with $R_{x}=6, R_{u}=14$. 

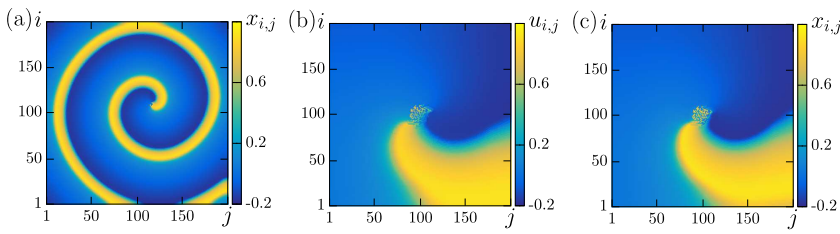

FIG. 8. Snapshots of (a) $x_{i j}$ in the first (response) lattice at $R_{x}=4, \gamma=0$, (b) $u_{i, j}$ in the second (driver) lattice at $R_{u}=22, \gamma=0$, and (c) $x_{i, j}$ in the response lattice for $\gamma=0.05$.

numerical simulations are shown in Figs. 8-11 for two different spiral wave chimera structures realized in the driver lattice. When the lattices are uncoupled, a spiral wave chimera is established in the second (driver) lattice [Fig. 8(b)], and a coherent spiral wave is realized in the first (response) lattice [Fig. 8(a)]. The partial external synchronization which takes place in the coupled lattices starting with $\gamma \geq$ 0.05 [Fig. 9(a)] results in the synchronous state shown in Fig. 8(c). However, as can be seen from Fig. 9(c), the oscillators in the incoherent core of the spiral wave chimera [in the center of the lattice in Fig. 8(c)] are desynchronized, and this feature is preserved for sufficiently large values of $\gamma \geq 0.2$ [Fig. 9(a)]. If the driver lattice exhibits a spatiotemporal structure with several incoherent cores, the number of desynchronized oscillators increases as shown for a five-core spiral wave chimera in Figs. 10(b) and 11(c). Moreover, they correspond exactly to the incoherent cores in the resulting synchronous pattern [Fig. 10(c)] and remain desynchronized even for a stronger unidirectional coupling strength [Fig. 11(a)]. Our numerical studies have shown that this effect is general for both mutual and external synchronizations. The number of desynchronized oscillators grows when the number of incoherent cores increases (compare Figs. 8-11 and 4-7). It has been found that there is a relation between the number of desynchronized oscillators in the case of the five-core spiral wave chimera $N_{5 \text { cores }}^{d}$ [Fig. 11(c)] and the single-core spiral wave chimera $N_{1 \text { core }}^{d}$, which are realized for the same parameters as in Fig. 11,

$$
N_{5 \text { cores }}^{d} \approx 5 N_{1 \text { core }}^{d}
$$

The imperfect synchronization occurs at a larger value of the unidirectional interlattice coupling $\gamma$ in comparison with the mutual synchronization [Figs. 5(b), 7(b), 9(b), and 11(b)].
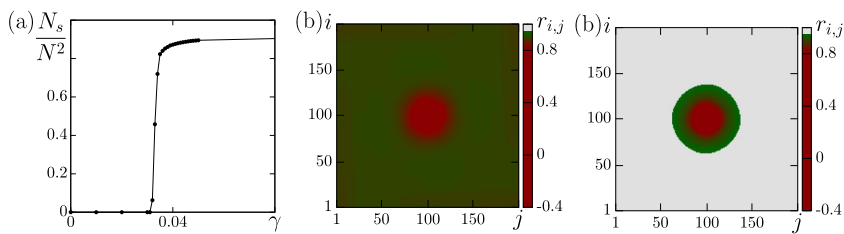

FIG. 9. (a) Number of synchronized oscillators $\left(\left\{N_{s}: r_{i, j}>0.95\right\}\right)$ vs the unidirectional interlattice coupling strength $\gamma$, distribution of the $r_{i, j}$ values at (b) $\gamma=0.02$ and (c) $\gamma=0.05$ with $R_{x}=4, R_{u}=22$.
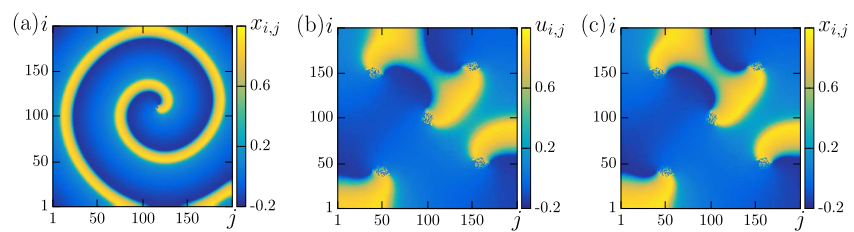

FIG. 10. Snapshots of (a) $x_{i j}$ in the first (response) lattice at $R_{x}=4, \gamma=0$, (b) $u_{i, j}$ in the second (driver) lattice at $R_{u}=14, \gamma=0$, and (c) $x_{i, j}$ in the response lattice at $\gamma=0.05$.

We have considered in detail the features of the dynamics of synchronized and desynchronized oscillators for the case of external synchronization illustrated in Fig. 8. In this case, the response lattice exhibiting a spiral wave [Fig. 8(a)] is unidirectionally affected by the driver lattice exhibiting a spiral wave chimera [Fig. 8(b)].

In order to estimate the degree of synchrony of oscillations, we calculate the Fourier spectra of the amplitudes of the corresponding oscillators [with the same numbers $(i, j)$ ] for the driver and response lattices (Fig. 12). Our calculations show that oscillators from regions of synchronous (coherent) dynamics are characterized by completely equivalent spectra [Fig. 12(a)]. However, Fourier spectra of the amplitudes for the oscillators from the incoherent region [the lattice center in Fig. 9(c)] have more peaks than the first ones and do not coincide for the same oscillators $(i, j)$ in both lattices [Fig. 12(b)]. The basic modes in the spectra differ in amplitude, whereas their harmonics coincide in frequency.

Our calculations have shown that for both mutual and unidirectional coupling, complete synchronization is not realized even when the coupling strength $\gamma$ and the computation time increase significantly. In the case of spiral wave chimeras observed in one of the lattices without coupling, an increase in mutual coupling strengths up to 1 can destroy the spatiotemporal structures, and the synchronization effect does not occur. The structures persist and are not destroyed in the case of external synchronization, but complete synchronization is also not achieved. All the structures observed in this work rotate as a whole with increasing calculation (observation) time. In the case of spiral wave chimeras, one part of the oscillators in the incoherent cores rotates together with the coherent cluster, while the other part behaves differently.
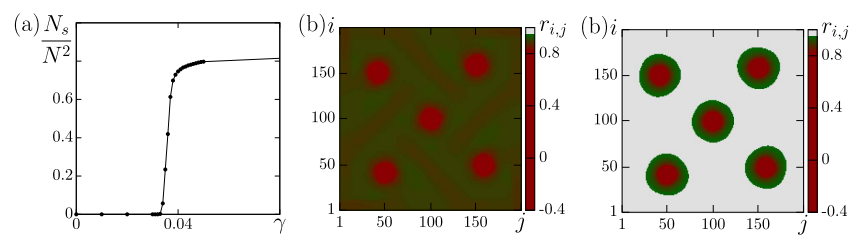

FIG. 11. (a) Number of synchronized oscillators $\left(\left\{N_{s}: r_{i j}>0.95\right\}\right)$ depending on the unidirectional interlattice coupling strength $\gamma$, distribution of the $r_{i, j}$ values at (b) $\gamma=0.02$ and (c) $\gamma=0.05$ with $R_{x}=4, R_{u}=14$. 

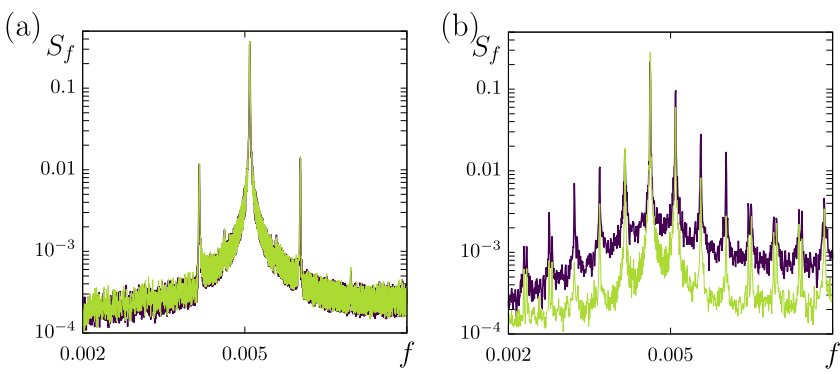

FIG. 12. Comparison of the Fourier spectra of the amplitudes $x_{i, j}$ (purple) and $u_{i, j}$ (green) of oscillators with (a) $i=100, j=50$ in coherent region and (b) $i=$ $100, j=105$ in incoherent core with $R_{x}=4, R_{u}=22, \gamma=0.05$.

\section{CONCLUSIONS}

In the present paper, we have analyzed numerically the mutual and external synchronization of two coupled lattices consisting of nonlocally coupled Nekorkin maps. Our numerical studies have shown that these synchronization effects are characterized by several important features. First, if each of the uncoupled lattices exhibits simple spiral wave structures, imperfect (almost complete) synchronization of oscillations of most corresponding elements of the two lattices can occur for a sufficient value of the coupling strength $\gamma$. We note that in the case of mutual synchronization, the resulting synchronous structure differs from the initially established modes in the uncoupled lattices. This effect is typical and has also been encountered when mutual synchronization of two oscillators with limit cycles ${ }^{85}$ was considered. Complete synchronization cannot be achieved even with a significant increase in the coupling strength, if one of the lattices exhibits a spiral wave chimera pattern for zero interlattice coupling. The second peculiarity consists of the fact that not all oscillators are almost completely synchronized when the lattices are coupled mutually or unidirectionally. A certain number of oscillators remain desynchronized, while most of them demonstrate imperfect (almost complete) synchronization. This effect takes place even in the case of simple (single core) initial patterns in both lattices and is enhanced when the initial spiral wave chimera in one of the interacting lattices has multiple cores.

\section{ACKNOWLEDGMENTS}

This work was funded by the Deutsche Forschungsgemeinschaft (DFG, German Research Foundation)_Projektnummer 163436311SFB 910 and by the Russian Ministry of Education and Science (Project Code 3.8616.2017).

\section{REFERENCES}

${ }^{1}$ L. M. Pecora, F. Sorrentino, A. M. Hagerstrom, T. E. Murphy, and R. Roy, Nat. Commun. 5, 4079 (2014)

${ }^{2}$ V. S. Afraimovich, V. I. Nekorkin, G. V. Osipov, and V. D. Shalfeev, Stability, Structures and Chaos in Nonlinear Synchronization Networks, World Scientific Series on Nonlinear Science Series A (World Scientific, 1995).

${ }^{3}$ V. I. Nekorkin, V. B. Kazantsev, and M. G. Velarde, Phys. Lett. A 236, 505-512 (1997).

${ }^{4}$ V. I. Nekorkin, M. L. Voronin, and M. G. Velarde, Eur. Phys. J. B 9, 533-543 (1999).
${ }^{5}$ V. N. Belykh, I. V. Belykh, and M. Hasler, Phys. Rev. E 62, 6332-6345 (2000).

${ }^{6}$ V. N. Belykh, I. V. Belykh, and E. Mosekilde, Phys. Rev. E 63, 036216 (2001).

${ }^{7}$ V. Nekorkin, V. Kazantsev, and M. Velarde, Physica D 151, 1-26 (2001).

${ }^{8}$ V. I. Nekorkin and M. G. Velarde, Synergetic Phenomena in Active Lattices, Springer Series in Synergetics (Springer, 2002).

${ }^{9}$ A. Akopov, V. Astakhov, T. Vadivasova, A. Shabunin, and T. Kapitaniak, Phys. Lett. A 334, 169-172 (2005).

${ }^{10}$ Y. Kuramoto, in Nonlinear Dynamics and Chaos. Where Do We Go from Here? edited by J. Hogan, A. Krauskopf, M. di Bernado, R. E. Wilson, H. M. Osinga, M. E. Homer, and A. R. Champneys (CRC Press, 2002), pp. 209-227.

${ }^{11}$ Y. Kuramoto and D. Battogtokh, Nonlinear Phenom. Complex Syst. 5, 380-385 (2002).

${ }^{12}$ D. M. Abrams and S. H. Strogatz, Phys. Rev. Lett. 93, 174102 (2004).

${ }^{13}$ M. J. Panaggio and D. M. Abrams, Nonlinearity 28, R67-R87 (2015).

${ }^{14}$ M. De Domenico, A. Solé-Ribalta, E. Cozzo, M. Kivelä, Y. Moreno, M. A. Porter, S. Gómez, and A. Arenas, Phys. Rev. X 3, 041022 (2013).

${ }^{15}$ M. Kivelä, A. Arenas, M. Barthelemy, J. P. Gleeson, Y. Moreno, and M. A. Porter, J. Complex Netw. 2, 203-271 (2014).

${ }^{16}$ S. Boccaletti, G. Bianconi, R. Criado, C. delGenio, J. Gómez-Gardeñes, M. Romance, I. Sendiña-Nadal, Z. Wang, and M. Zanin, Phys. Rep. 544, 1-122 (2014).

${ }^{17}$ L. V. Gambuzza, M. Frasca, and J. Gómez-Gardeñes, Europhys. Lett. 110, 20010 (2015).

${ }^{18}$ S. Ghosh, A. Kumar, A. Zakharova, and S. Jalan, Europhys. Lett. 115, 60005 (2016).

${ }^{19}$ R. Sevilla-Escoboza, I. Sendiña-Nadal, I. Leyva, R. Gutiérrez, J. M. Buldú, and S. Boccaletti, Chaos 26, 065304 (2016).

${ }^{20}$ S. Ghosh and S. Jalan, Int. J. Bifurc. Chaos 26, 1650120 (2016).

${ }^{21}$ V. A. Maksimenko, V. V. Makarov, B. K. Bera, D. Ghosh, S. K. Dana, M. V. Goremyko, N. S. Frolov, A. A. Koronovskii, and A. E. Hramov, Phys. Rev. E 94, 052205 (2016).

${ }^{22}$ M. Mikhaylenko, L. Ramlow, S. Jalan, and A. Zakharova, Chaos 29, 023122 (2019).

${ }^{23}$ R. G. Andrzejak, G. Ruzzene, and I. Malvestio, Chaos 27, 053114 (2017).

${ }^{24}$ A. Singh, S. Ghosh, S. Jalan, and J. Kurths, Europhys. Lett. 111, 30010 (2015).

${ }^{25}$ S. Boccaletti, J. Almendral, S. Guan, I. Leyva, Z. Liu, I. Sendiña-Nadal, Z. Wang, and Y. Zou, Phys. Rep. 660, 1-94 (2016).

${ }^{26}$ A. Bukh, E. Rybalova, N. Semenova, G. Strelkova, and V. Anishchenko, Chaos 27, 111102 (2017).

${ }^{27}$ A. V. Bukh, G. I. Strelkova, and V. S. Anishchenko, Russ. J. Nonlin. Dyn. 14, 419-433 (2018).

${ }^{28}$ G. Strelkova, T. Vadivasova, and V. Anishchenko, Regul. Chaotic Dyn. 23, 948-960 (2018).

${ }^{29}$ E. Rybalova, T. Vadivasova, G. Strelkova, V. Anishchenko, and A. Zakharova, Chaos 29, 033134 (2019).

${ }^{30}$ I. Leyva, R. Sevilla-Escoboza, I. SendiaNadal, R. Gutiérrez, J. Buldú, and S. Boccaletti, Sci. Rep. 7, 45475 (2017).

${ }^{31}$ J. Sawicki, I. Omelchenko, A. Zakharova, and E. Schöll, Phys. Rev. E 98, 062224 (2018).

${ }^{32}$ X. Zhang, S. Boccaletti, S. Guan, and Z. Liu, Phys. Rev. Lett. 114, 038701 (2015).

${ }^{33}$ I. Leyva, I. Sendiña-Nadal, R. Sevilla-Escoboza, V. P. Vera-Avila, P. Chholak, and S. Boccaletti, Sci. Rep. 8, 8629 (2018).

${ }^{34}$ I. Leyva, I. Sendiña-Nadal, and S. Boccaletti, Discrete Continuous Dyn. Syst. Ser. B 25, 1931 (2018).

${ }^{35}$ A. D. Kachhvah and S. Jalan, New J. Phys. 21, 015006 (2019).

${ }^{36}$ I. Omelchenko, Y. Maistrenko, P. Hövel, and E. Schöll, Phys. Rev. Lett. 106, 234102 (2011).

${ }^{37}$ I. Omelchenko, B. Riemenschneider, P. Hövel, Y. Maistrenko, and E. Schöll, Phys. Rev. E 85, 026212 (2012).

${ }^{38}$ A. M. Hagerstrom, T. E. Murphy, R. Roy, P. Hövel, I. Omelchenko, and E. Schöll, Nat. Phys. 8, 658-661 (2012).

${ }^{39}$ M. R. Tinsley, S. Nkomo, and K. Showalter, Nat. Phys. 8, 662-665 (2012).

${ }^{40}$ L. Larger, B. Penkovsky, and Y. Maistrenko, Phys. Rev. Lett. 111, 054103 (2013).

${ }^{41}$ E. A. Martens, S. Thutupalli, A. Fourrière, and O. Hallatschek, Proc. Natl. Acad.

Sci. U.S.A. 110, 10563-10567 (2013).

${ }^{42}$ M. J. Panaggio and D. M. Abrams, Phys. Rev. E 110, 094102 (2013). 
${ }^{43}$ D. Dudkowski, Y. Maistrenko, and T. Kapitaniak, Phys. Rev. E 90, 032920 (2014).

${ }^{44}$ Y. L. Maistrenko, A. Vasylenko, O. Sudakov, R. Levchenko, and V. L. Maistrenko, Int. J. Bifurc. Chaos 24, 1440014 (2014).

${ }^{45}$ A. Yeldesbay, A. Pikovsky, and M. Rosenblum, Phys. Rev. Lett. 112, 144103 (2014).

${ }^{46}$ T. Kapitaniak, P. Kuzma, J. Wojewoda, K. Czolczynski, and Y. Maistrenko, Sci. Rep. 4, 6379 (2014).

${ }^{47}$ J. Hizanidis, E. Panagakou, I. Omelchenko, E. Schöll, P. Hövel, and A. Provata, Phys. Rev. E 92, 012915 (2015).

${ }^{48}$ N. Semenova, A. Zakharova, E. Schöll, and V. Anishchenko, Europhys. Lett. 112, 40002 (2015).

${ }^{49}$ S. Olmi, E. A. Martens, S. Thutupalli, and A. Torcini, Phys. Rev. E 92, 030901 (2015).

${ }^{50}$ M. J. Panaggio and D. M. Abrams, Phys. Rev. E 91, 022909 (2015).

${ }^{51}$ F. P. Kemeth, S. W. Haugland, L. Schmidt, I. G. Kevrekidis, and K. Krischer, Chaos 26, 094815 (2016).

${ }^{52}$ E. Schöll, Eur. Phys. J. Spec. Top. 225, $891-919$ (2016).

${ }^{53}$ S. Ulonska, I. Omelchenko, A. Zakharova, and E. Schöll, Chaos 26, 094825 (2016).

${ }^{54}$ N. Semenova, A. Zakharova, V. Anishchenko, and E. Schöll, Phys. Rev. Lett. 117, 014102 (2016).

${ }^{55}$ V. Semenov, A. Zakharova, Y. Maistrenko, and E. Schöll, Europhys. Lett. 115, 10005 (2016).

${ }^{56}$ J. Hizanidis, N. E. Kouvaris, G. Zamora-Lòpez, A. Dìaz-Guilera, and C. G. Antonopoulos, Sci. Rep. 6, 19845 (2016).

${ }^{57}$ S. Majhi, M. Perc, and D. Ghosh, Sci. Rep. 6, 39033 (2016).

${ }^{58}$ J. Sawicki, I. Omelchenko, A. Zakharova, and E. Schöll, Eur. Phys. J. Spec. Top. 226, 1883-1892 (2017).

${ }^{59}$ E. Rybalova, N. Semenova, G. Strelkova, and V. Anishchenko, Eur. Phys. J. Spec. Top. 226, 1857-1866 (2017)

${ }^{60}$ S. A. Bogomolov, A. V. Slepnev, G. I. Strelkova, E. Schöll, and V. S. Anishchenko, Commun. Nonlinear Sci. Numer. Simul. 43, 25-36 (2016).

${ }^{61}$ C.-H. Tian, X.-Y. Zhang, Z.-H. Wang, and Z.-H. Liu, Front. Phys. 12, 128904 (2017).

${ }^{62}$ A. Schmidt, T. Kasimatis, J. Hizanidis, A. Provata, and P. Hövel, Phys. Rev. E 95, 032224 (2017).
${ }^{63}$ I. Shepelev, A. Bukh, T. Vadivasova, V. Anishchenko, and A. Zakharova, Commun. Nonlinear Sci. Numer. Simul. 54, 50-61 (2018).

${ }^{64}$ M. Hildebrand, J. Cui, E. Mihaliuk, J. Wang, and K. Showalter, Phys. Rev. E 68, 026205 (2003).

${ }^{65}$ Y. Kuramoto and S.-I. Shima, Prog. Theor. Phys. Suppl. 150, 115-125 (2003).

${ }^{66}$ S.-I. Shima and Y. Kuramoto, Phys. Rev. E 69, 036213 (2004).

${ }^{67}$ E. A. Martens, C. R. Laing, and S. H. Strogatz, Phys. Rev. Lett. 104, 044101 (2010).

${ }^{68} \mathrm{X}$. Tang, T. Yang, I. Epstein, Y. Liu, Y. Zhao, and Q. Gao, J. Chem. Phys. 141, 024110 (2014).

${ }^{69}$ J. Xie, E. Knobloch, and H.-C. Kao, Phys. Rev. E 92, 042921 (2015).

${ }^{70}$ J. F. Totz, J. Rode, M. R. Tinsley, K. Showalter, and H. Engel, Nat. Phys. 14, 282-285 (2018).

${ }^{71}$ C. R. Laing, Physica D 238, 1569-1588 (2009).

${ }^{72}$ S. Nkomo, M. R. Tinsley, and K. Showalter, Phys. Rev. Lett. 110, 244102 (2013).

${ }^{73}$ C. Gu, G. St-Yves, and J. Davidsen, Phys. Rev. Lett. 111, 134101 (2013).

${ }^{74}$ Y. Kuramoto, S.-I. Shima, D. Battogtokh, and Y. Shiogai, Prog. Theor. Phys. Suppl. 161, 127-143 (2006).

${ }^{75}$ B.-W. Li and H. Dierckx, Phys. Rev. E 93, 020202 (2016).

${ }^{76}$ S. Weiss and R. D. Deegan, Phys. Rev. E 95, 022215 (2017).

${ }^{77}$ S. Kundu, S. Majhi, P. Muruganandam, and D. Ghosh, Eur. Phys. J. Spec. Top. 227, 983-993 (2018).

${ }^{78}$ S. Guo, Q. Dai, H. Cheng, H. Li, F. Xie, and J. Yang, Chaos Solitons Fractals 114, 394-399 (2018).

${ }^{79}$ A. Bukh, G. Strelkova, and V. Anishchenko, Chaos Solitons Fractals 120, 75-82 (2019).

${ }^{80} \mathrm{~V}$. Nekorkin and L. Vdovin, Prikladnaya nelineynaya dinamika (Appl. Nonlinear Dyn.) 15, 36-60 (2007).

${ }^{81}$ E. M. Izhikevich, IEEE Trans. Neural Netw. 15, 1063-1070 (2004).

${ }^{82}$ A. Pikovsky and A. Politi, Lyapunov Exponents (Cambridge University Press, 2016).

${ }^{83}$ S. W. Haugland, L. Schmidt, and K. Krischer, Sci. Rep. 5, 9883 (2015).

${ }^{84}$ T. E. Vadivasova, G. I. Strelkova, S. A. Bogomolov, and V. S. Anishchenko, Chaos 26, 093108 (2016).

${ }^{85}$ A. Pikovsky, M. Rosenblum, and J. Kurths, Synchronization: A Universal Concept in Nonlinear Sciences, Cambridge Nonlinear Science Series (Cambridge University Press, 2003). 